\title{
Initial rapidity of tumor growth as a prognostic factor for the therapeutic effect of immune-checkpoint inhibitors in patients with non-small cell lung cancer: evaluation for linear and non-linear correlation
}

\author{
Kosuke Sakai $^{1,2}$, Joji Kuramoto ${ }^{1}$, Hiroaki Nishimura ${ }^{1}$, Yoshiki Kuwabara ${ }^{1}$, Akitoshi Kojima ${ }^{1}$, \\ Maiko Sasaki-Toda ${ }^{1}$, Yumiko Ogawa-Kobayashi ${ }^{1,2}$, Satoshi Kikuchi ${ }^{1}$, Yusuke Hirata ${ }^{1}$, \\ Yuriko Mikami-Saito ${ }^{1}$, Shintaro Mikami ${ }^{1}$, Hiroyuki Kyoyama ${ }^{1}$, Gaku Moriyama ${ }^{1,2}$, Akihiko Gemma ${ }^{2}$, \\ Kazutsugu Uematsu ${ }^{1 \wedge}$ \\ ${ }^{1}$ Department of Pulmonary Medicine, Saitama Medical Center, Saitama Medical University, 1981 Kamoda, Kawagoe, Saitama 350-8550, Japan; \\ ${ }^{2}$ Department of Pulmonary Medicine and Oncology, Graduate School of Medicine, Nippon Medical School, 1-1-5 Sendagi, Bunkyo-ku, Tokyo 113- \\ 8603, Japan \\ Contributions: (I) Conception and design: K Sakai; (II) Administrative support: A Gemma, K Uematsu; (III) Provision of study materials or patients: \\ K Sakai, H Nishimura, Y Kuwabara, A Kojima, M Sasaki-Toda, Y Ogawa-Kobayashi, S Kikuchi, Y Hirata, Y Mikami-Saito, S Mikami, H Kyoyama, \\ G Moriyama, A Gemma, K Uematsu; (IV) Collection and assembly of data: K Sakai, J Kuramoto; (V) Data analysis and interpretation: K Sakai, K \\ Uematsu; (VI) Manuscript writing: All authors; (VII) Final approval of manuscript: All authors. \\ Correspondence to: Kazutsugu Uematsu, MD, PhD. Saitama Medical Center, Saitama Medical University, 1981 Kamoda, Kawagoe, Saitama 350-8550, \\ Japan. Email: kuematsu@saitama-med.ac.jp.
}

Background: Immune-checkpoint inhibitors (ICIs) have been increasingly used for non-small cell lung cancer (NSCLC) treatment in recent years. Although insufficient, the rate of programmed death-ligand 1 expression has been adopted as a predictor of ICI efficacy. We evaluated tumor growth rate as a clinically easy-to-use predictor of the therapeutic effect of ICIs.

Methods: This study is a single-institution retrospective study in Japan. NSCLC patients treated with nivolumab, pembrolizumab, or atezolizumab at Saitama Medical Center from January 1, 2016 to December 31, 2018 were enrolled, and followed until December 31, 2020. We defined and calculated the initial rapidity of tumor progression (IRP) as: the increase in the sum of the diameters of intrathoracic tumors and lymph nodes on two series of chest computed tomography (CT) scans (one obtained at an initial checkup and the other obtained immediately before the first treatment) divided by the number of days between these CT scans. Two coefficients were calculated: the maximal information coefficient (MIC) between IRP and time to treatment failure (TTF) using the Python package with minepy library, and the Spearman's rank correlation coefficient.

Results: A total of 55 patients (median age, 70 years; 47 men) were enrolled. The median TTF with ICIs was 126 days, and four patients continued to receive ICI treatment at the end of the follow-up. The MIC between IRP and TTF was 0.302 with weak correlation, and the Spearman's rank correlation coefficient was $-0.347(\mathrm{P}=0.00938)$.

Conclusions: The initial tumor growth rate had a negative linear correlation with the therapeutic effect of ICIs.

Keywords: Immune-checkpoint inhibitors (ICIs); predictor; growth rate of tumor; maximal information coefficient

^ ORCID: Kosuke Sakai, 0000-0003-3138-0360; Kazutsugu Uematsu, 0000-0003-0014-295X. 
Submitted May 04, 2021. Accepted for publication Jul 23, 2021.

doi: $10.21037 /$ jtd-21-774

View this article at: https://dx.doi.org/10.21037/jtd-21-774

\section{Introduction}

Lung cancer is the leading cause of cancer-related death, and its prevalence is increasing worldwide. The prevalence of tracheal, bronchus, and lung cancer increased by $28 \%$ from 2006 to 2016; 1.7 million patients died in 2016 (1). Lung cancer is frequently detected in an already advanced stage; therefore, most patients have no choice but chemotherapy as the primary therapy. Unfortunately, the prognosis of lung cancer patients with distant metastasis is devastating, and their median overall survival at our hospital was 9.5 months (2).

Docetaxel has hitherto been the standard secondline chemotherapy (3); however, CheckMate-017 and CheckMate-057 revealed that nivolumab, the firstreleased immune-checkpoint inhibitor (ICI), prolonged the overall survival compared with docetaxel $(4,5)$. Afterward, pembrolizumab was approved as the first-line therapy against non-small cell lung cancer (NSCLC) with programmed deathligand 1 (PD-L1) expression of 50\% or higher (6). However, at present, ICIs in combination with cytotoxic chemotherapies have been approved as the first-line treatment for lung cancer regardless of PD-L1 expression $(7,8)$.

Clinically useful predictors of ICI treatment efficacy are needed. Other biomarkers besides PD-L1 expression rate have also been proposed as candidates. Tumor mutation burden detected by next-generation sequencing has been reported to correlate with ICI treatment efficacy (9); however, its application is clinically limited, and a standard value remains to be set. Microsatellite instability is the first cross-organ biomarker approved by the U.S. Food and Drug Administration (10); however, its clinical efficacy has not been proven for NSCLC. The effectiveness of peripheral blood CD62L ${ }^{\text {low }} \mathrm{CD}^{+}{ }^{+} \mathrm{T}$-cells as indicators of therapeutic response to ICIs is examined experimentally (11). At present, more convenient predictors are still necessary. The baseline tumor diameter of more than $101 \mathrm{~mm}$ has been shown to predict poor prognosis (12). We anticipated that tumor growth rate, which represents a change of tumor diameter over a period of time, might relate to prognosis with ICI treatment.

In this study, to evaluate the tumor growth rate during the pre-treatment period as a predictor of ICIs efficacy, we scatterplotted initial rapidity of the tumor growth as a function of time to treatment failure (TTF) and then calculated non-linear and linear coefficients. We adopted the maximal information coefficient (MIC), which is a novel non-linear correlation coefficient (13), and the conventional Spearman's rank correlation coefficient as estimators of linear correlation.

We present the following article in accordance with the STROBE reporting checklist (available at https://dx.doi. org/10.21037/jtd-21-774).

\section{Methods}

\section{Patients}

All NSCLC patients, who had undergone nivolumab, pembrolizumab, or atezolizumab therapy at Saitama Medical Center, Saitama Medical University from January 1, 2016 to December 31, 2018 were retrospectively enrolled. For measurement of the tumor growth rate during the pre-treatment period and the best response to ICIs, chest computed tomography (CT) needs to be performed at least twice before the start of ICI treatment, and at least once after the treatment. Patients who did not undergo these three chest CT examinations were excluded. Follow-up was continued until December 31, 2020.

\section{Procedures}

Clinical information was retrospectively collected from electronic medical records at Saitama Medical Center. We defined a tumor size as the sum of the diameters of the intrathoracic tumors and the lymph nodes on chest CT images, and an initial tumor size (ITS) was obtained at the first checkup in a previous hospital or clinic, or at Saitama Medical Center. We also defined the initial rapidity of tumor progression (IRP) as the calculated increase in the summed diameter from the time of the first CT scan (initial checkup) to the time of the second CT scan (just before initiation of treatment) divided by the number of days between the two scans. After intrathoracic tumor lesions were detected, lung lesions of $10 \mathrm{~mm}$ or more in a major axis and lymph nodes of $15 \mathrm{~mm}$ or more in a minor axis were defined as measurable lesions, and the top two largest lesions each in lung lesions and lymph nodes were measured 
A

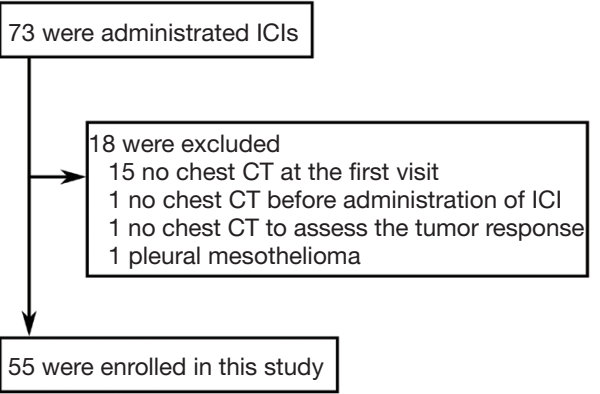

B

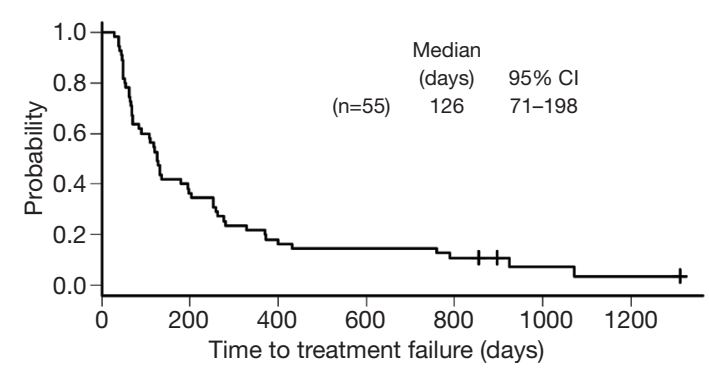

C

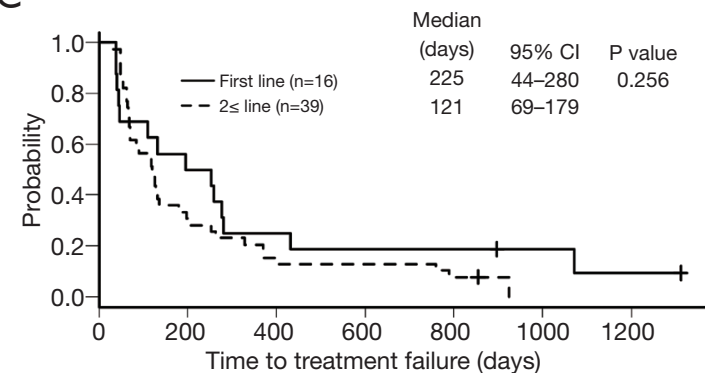

Figure 1 Patient selection and time to treatment failure (TTF). (A) Flow diagram of patient selection. (B) A Kaplan-Meier curve of TTF in all enrolled patients who underwent ICI treatment. (C) Kaplan-Meier curves of TTF by ICI administered as firstline treatment (-) and second- or more-line treatment (--). ICI, immune-checkpoint inhibitor; CT, computed tomography; CI, confidence interval.

for IRP calculation. For response evaluation, up to 5 lesions including other organ lesions were measured. A specialized physician in respiratory diseases and a data management assistant measured lesions according to the RECIST guideline version 1.1. TTF was defined as an interval from ICI treatment initiation to its withdrawal by an attending physician. We also accumulated their neutrophil counts, lymphocyte counts, the value of C-reactive protein (CRP), and the value of albumin on their first visit, and calculated the initial value of the neutrophil-lymphocyte ratio (NLR) and the CRP-albumin ratio. We examined the correlation of ITS, IRP, the initial NLR and the initial CRP-albumin ratio with TTF to verify the tumor growth rate as a predictor of ICI benefits.

\section{Statistics}

TTF was estimated by the Kaplan-Meier method, and the differences between groups were evaluated by the log-rank test using the EZR software program (Saitama Medical Center, Jichi Medical University, Saitama, Japan) (14), with the level of significance set at $\mathrm{P}<0.05$. A Normal distribution of patients' parameters was verified by the Shapiro-Wilk test, and scatter plots were drawn and analyzed to determine correlation. Three or more groups were compared with a non-parametric multiple test, the Kruskal-Wallis test. Spearman's rank correlation coefficient and MIC were adopted as tools for use in distribution analysis on scatter plots with EZR. MIC was calculated using the tools implemented in Python 3.6.5 with the imported minepy library. Spearman's rank correlation coefficient with $\mathrm{P}<0.05$ was considered statistically significant, and the absolute value of their coefficients was interpreted as the follows: $0-0.3$, no correlation; $0.3-0.5$, weak correlation; $0.5-0.7$, moderate correlation; $0.7-0.9$, high correlation; and 0.9-1.0, very high correlation. MIC was evaluated according to interpretation for Spearman's rank correlation coefficients.

\section{Ethical statement}

The study was conducted in accordance with the Declaration of Helsinki (as revised in 2013). The study was approved by the ethics board of Saitama Medical Center, Saitama Medical University (application number: 2179) and individual consent for this retrospective analysis was waived.

\section{Results}

A total of 73 patients underwent ICI treatment at Saitama Medical Center; however, 18 patients were excluded from this study because of insufficient chest CT information in 17 patients and a different diagnosis of mesothelioma in 1 patient (Figure 1A).

A total of 55 patients were included [ 47 men and 8 women, 44-88 years old (median age, 70 years old)]; 29 had pembrolizumab, 16 first-line treatment, and 13 secondor more-line treatment; 22 had nivolumab, and 4 had atezolizumab for second- or more-line treatment (Table 1). The 
Table 1 Characteristics of patients

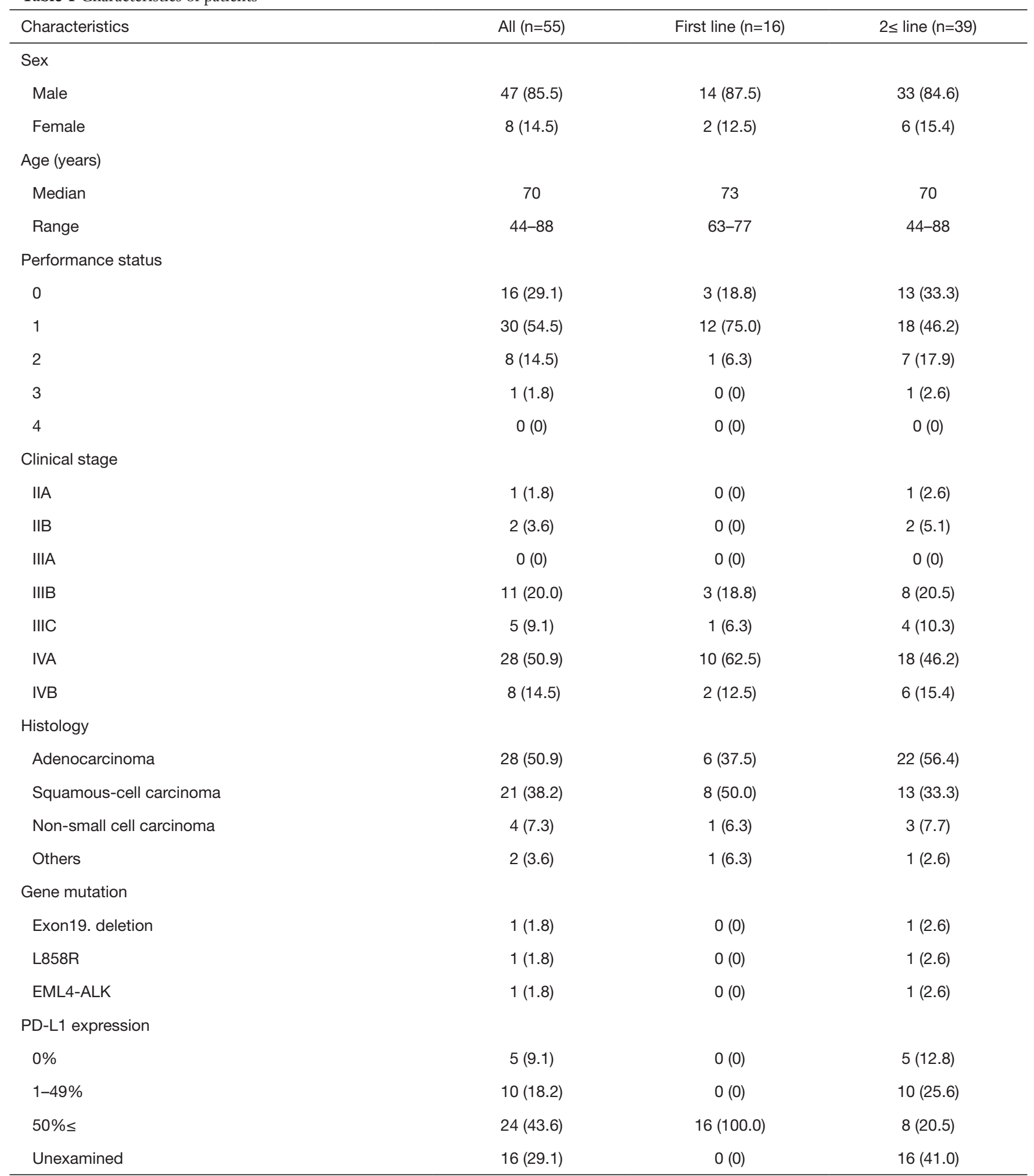

Table 1 (continued) 
Table 1 (continued)

\begin{tabular}{|c|c|c|c|}
\hline Characteristics & All $(n=55)$ & First line $(n=16)$ & $2 \leq$ line $(n=39)$ \\
\hline \multicolumn{4}{|l|}{ Administrated ICls } \\
\hline Nivolumab & $22(40.0)$ & $0(0)$ & $22(56.4)$ \\
\hline Pembrolizumab & $29(52.7)$ & $16(100.0)$ & $13(33.3)$ \\
\hline Atezolizumab & $4(7.3)$ & $0(0)$ & $4(10.3)$ \\
\hline Complete response & $5(9.1)$ & $3(18.8)$ & $2(5.1)$ \\
\hline Partial response & $14(25.5)$ & 5 (31.3) & $9(23.1)$ \\
\hline Stable disease & $27(49.1)$ & $5(31.3)$ & $22(56.4)$ \\
\hline Progressive disease & $9(16.4)$ & $3(18.8)$ & $6(15.4)$ \\
\hline
\end{tabular}

PD-L1, programmed death-ligand 1; ICls, immune-checkpoint inhibitors.

Table 2 Reasons for discontinuing immune-checkpoint inhibitors

\begin{tabular}{|c|c|c|c|}
\hline Reasons for discontinuing & All $(n=55)$ & First line $(n=16)$ & $2 \leq$ line $(n=39)$ \\
\hline Pneumonia & $9(16.4)$ & $2(12.5)$ & $7(17.9)$ \\
\hline Liver function disorder & $1(1.8)$ & $0(0)$ & $1(2.6)$ \\
\hline Hypothyroidism & $2(3.6)$ & $0(0)$ & $2(5.1)$ \\
\hline Peripheral neuropathy & $1(1.8)$ & $0(0)$ & $1(2.6)$ \\
\hline Skin rash & $1(1.8)$ & $1(6.3)$ & $0(0)$ \\
\hline Others & $2(3.6)$ & $2(12.5)$ & $0(0)$ \\
\hline Continuing & $4(7.3)$ & $2(12.5)$ & $2(5.1)$ \\
\hline
\end{tabular}

best confirmed response to ICIs was: complete response (CR), 5 (9.1\%); partial response (PR), 14 (25.5\%); stable disease (SD), 27 (49.1\%); and progressive disease (PD), 9 (16.4\%).

While four patients continued ICI treatments over the follow-up period, 51 patients were withdrawn from treatment due to the following: tumor progression, 34; pneumonia, 9; hypothyroidism, 2; liver function disorder, 1; hypophysitis, 1; peripheral neuropathy, 1; and skin rash, 1 (Table 2).

The median TTF of ICI treatment for all 55 patients was 126 days (range, 28-1,311 days) (Figure 1B). TTF for 16 patients with the first-line ICI was not significantly different from that for 39 patients with the second- or moreline ICI ( $\mathrm{P}=0.256)$, while the former tended to be longer than the latter, with the median TTF, 225 days (38-1,311 days) and 121 days (28-925 days), respectively (Figure 1C).

The median interval between the first CT scan at the initial checkup and the second CT scan just before treatment was 44 days (range, 5-1,573 days), and the median IRP during the period was $0.14 \mathrm{~mm} /$ days $(0.02-2.09 \mathrm{~mm} /$ days $)$. Twenty-five patients had extrathoracic metastases before the first treatment, and the median sum of diameters in each metastatic site was the following: the brain $(\mathrm{n}=10), 18.75 \mathrm{~mm}$ (range, $3.7-38.4 \mathrm{~mm})$; the bone $(\mathrm{n}=12), 26.15 \mathrm{~mm}$ $(3.5-86.0 \mathrm{~mm})$; the liver $(\mathrm{n}=2), 20.2$ and $25.1 \mathrm{~mm}$; the adrenal gland $(\mathrm{n}=4), 20.15 \mathrm{~mm}(17.4-39.4 \mathrm{~mm})$; the extrathoracic lymph node $(\mathrm{n}=2), 10.2 \mathrm{~mm}$ and $34.3 \mathrm{~mm}$; and the muscle $(\mathrm{n}=1), 8.4 \mathrm{~mm}$. A patient with a $38.4-\mathrm{mm}$ nodule 
Table 3 Correlation coefficients between two factors

\begin{tabular}{|c|c|c|c|c|}
\hline Factor 1 & Factor 2 & $\begin{array}{c}\text { Maximal } \\
\text { information } \\
\text { coefficient }\end{array}$ & \multicolumn{2}{|c|}{ Spearman's rank correlation coefficient } \\
\hline Initial rapidity of tumor progression & Initial tumor size & 0.326 & 0.393 & 0.00296 \\
\hline Time to treatment failure & Initial rapidity of tumor progression & 0.302 & -0.347 & 0.00938 \\
\hline Time to treatment failure & Initial CRP-albumin ratio & 0.260 & -0.084 & 0.54300 \\
\hline
\end{tabular}

NLR, neutrophil-lymphocyte ratio; CRP, C-reactive protein.

in the cerebellum underwent tumor resection before ICI administration, and recurrence did not occur in the brain during the second-line pembrolizumab treatment. A patient with an 86-mm bulky mass in the ilium underwent radiation therapy, which apparently shrunk the bone mass, leading to osteosclerosis without fluorodeoxyglucose accumulation. While the patient received nivolumab treatment, recurrence did not occur in the bone.

We assumed that the correlation of IRP or ITS with TTF might not be linear; therefore, we examined the correlation of IRP and TTF using MICs, non-linear correlation coefficients reported for the first time by Reshef et al. in 2011 (13), and Spearman's rank correlation coefficients (Table 3). In a linear function set, MIC is 1 and is the same as Pearson's correlation coefficient; in a quadratic function, Pearson's correlation coefficient was 0.499 , and MIC was 1 ; and, in the inverse proportion correlation, Pearson's correlation coefficient was -0.648 , and MIC was 1 , indicating that MIC is a measure of the strength of non-linear correlation (Figure $2 A$ ). Since the Gompertzian model originally showed that the tumor growth rate slows as the tumor size increases (15), IRP was thought to be negatively correlated with ITS. However, Spearman's rank correlation coefficient between them was $0.393(\mathrm{P}=0.00296)$ indicating a weakly positive correlation (Figure $2 B$ ). The correlation of IRP to TTF was weak with MIC, 0.302, and negative linear, $-0.347(\mathrm{P}=0.00938)$, with Spearman's rank correlation coefficient (Figure 2C). On the other hand, ITS and TTF were not correlated using MIC, 0.259 , and Spearman's rank correlation coefficient, -0.228 $(\mathrm{P}=0.09430)$ (Figure 2D).

IRP was evaluated according to histology of lung cancer or PD-L1 expression. The median IRP in each histology of lung cancer is the following: $0.125 \mathrm{~mm} /$ days (range
$0.02-0.93)$ in adenocarcinoma, $0.115 \mathrm{~mm} /$ days $(0.04-1.17)$ in NSCLC, which was impossible to be determined as detailed types, $0.270 \mathrm{~mm} /$ days $(0.03-2.09)$ in squamous cell carcinoma, and $0.15 \mathrm{~mm} /$ days and $0.09 \mathrm{~mm} /$ days in the other two types $(\mathrm{P}=0.393)$ (Figure $3 A)$; therefore, IRP was not associated with histology of lung cancer. IRP and PDL1 expression were also not correlated in 39 patients, in which PD-L1 expression had been measured; MIC, 0.234; and Spearman's rank correlation coefficient, $0.111(\mathrm{P}=0.502)$ (Figure 3B).

The relationship between IRP and the response to treatment after 3, 6, and 9 months after ICI administration was evaluated (Figure 4). The median IRP in each response to treatment were the following: $\mathrm{CR},(\mathrm{n}=3), 0.18 \mathrm{~mm}$ /days (range $0.02-0.20 \mathrm{~mm} / \mathrm{days}) ;$ PR $(\mathrm{n}=16), 0.13 \mathrm{~mm} / \mathrm{days}$ $(0.03-1.17 \mathrm{~mm} /$ days $) ; \mathrm{SD}(\mathrm{n}=18), 0.085 \mathrm{~mm} /$ days $(0.02-0.45 \mathrm{~mm} /$ days); and discontinuation of ICI $(\mathrm{n}=15)$, $0.3 \mathrm{~mm} /$ days $(0.09-2.09 \mathrm{~mm} /$ days $)$ at three months after ICI administration $(\mathrm{P}=0.0217)$; $\mathrm{CR}(\mathrm{n}=2), 0.11 \mathrm{~mm} /$ days $(0.04-0.18 \mathrm{~mm} / \mathrm{days}), P R(\mathrm{n}=9), 0.32 \mathrm{~mm} / \mathrm{days}$ $(0.02-1.17 \mathrm{~mm} / \mathrm{days}) ; \mathrm{SD}(\mathrm{n}=9), 0.08 \mathrm{~mm} / \mathrm{days}$ $(0.02-0.45 \mathrm{~mm} / \mathrm{days}) ; \mathrm{PD}(\mathrm{n}=3), 0.09 \mathrm{~mm} / \mathrm{days}$ $(0.02-0.20 \mathrm{~mm} /$ days $)$; and discontinuation of ICI $(\mathrm{n}=31)$, $0.22 \mathrm{~mm} /$ days $(0.03-2.09 \mathrm{~mm} /$ days $)$ at six months $(\mathrm{P}=0.24)$; and CR ( $\mathrm{n}=3), 0.04 \mathrm{~mm} /$ days $(0.02-0.18 \mathrm{~mm} /$ days); $\mathrm{PR}(\mathrm{n}=5)$, $0.13 \mathrm{~mm} /$ days $(0.05-0.39 \mathrm{~mm} /$ days $) ; \mathrm{SD}(\mathrm{n}=5), 0.06 \mathrm{~mm} /$ days $(0.02-0.12 \mathrm{~mm} /$ days $)$; PD $(\mathrm{n}=4), 0.565 \mathrm{~mm} /$ days $(0.33-1.17 \mathrm{~mm} /$ days $)$; and discontinuation of ICI $(\mathrm{n}=36)$, $0.2 \mathrm{~mm} /$ days $(0.02-2.09 \mathrm{~mm} /$ days $)$ after nine months $(\mathrm{P}=0.00834)$. Due to lack of appropriate examination, 3, 1, and 2 patients were excluded from the evaluation at 3,6 , and 9 months, respectively.

In addition, the initial value of NLR or the CRP-albumin ratio, which has already been reported as a prognostic factor 
A

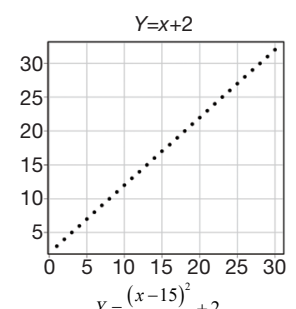

Pearson $=1$

$\mathrm{MIC}=1.000$

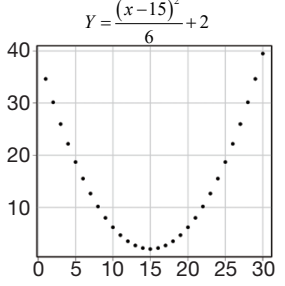

$Y=30 / x$

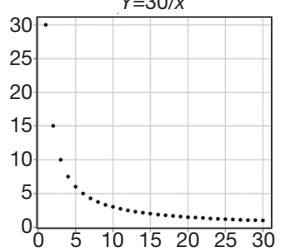

Pearson $=-0.648$

$\mathrm{MIC}=1.000$
C

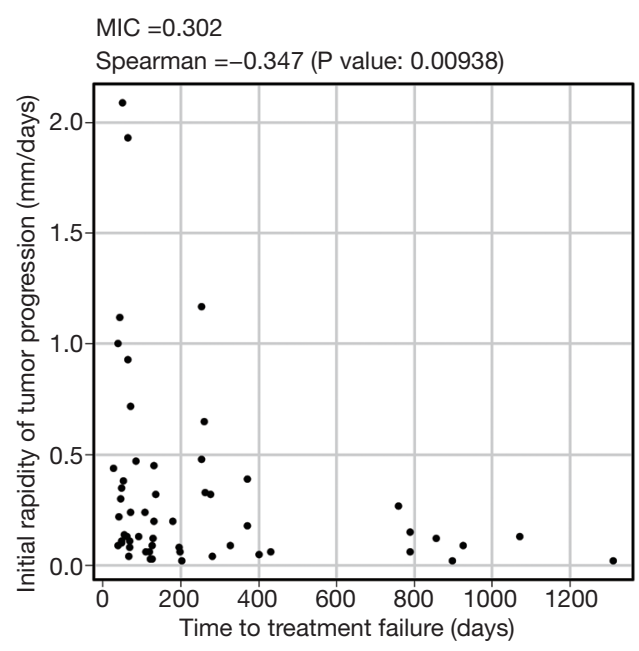

D $\quad$ MIC $=0.259$

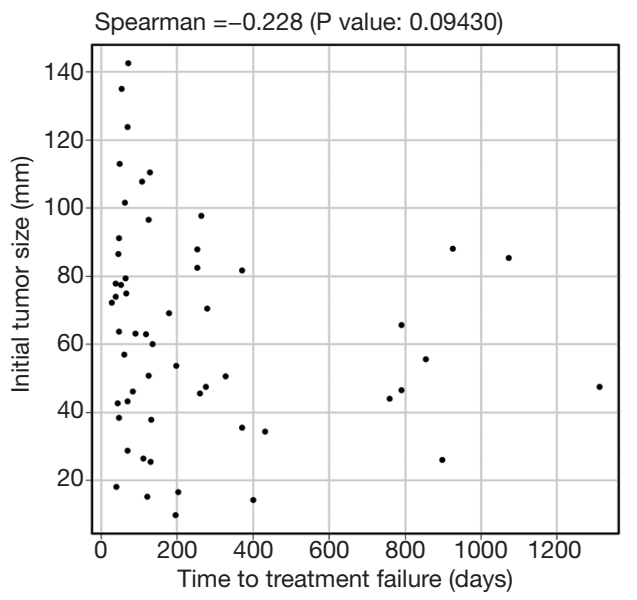

Figure 2 Scatter plots. (A) Comparison of Pearson's correlation coefficient (Pearson) and maximal information coefficient (MIC). (B) Correlation between initial rapidity of tumor progression and initial tumor size. (C) Correlation between initial rapidity of tumor progression and time to treatment failure (TTF). (D) Correlation between initial tumor size and TTF. Spearman, Spearman's rank correlation coefficient.

of treatment with ICIs $(16,17)$, was also examined. Although NLR has previously been reported as a negative predictor of ICI therapy, the initial NLR seemed to be positively correlated with TTF on the scatter plot, with MIC, 0.315; however, NLR did not linearly correlate with TTF using Spearman's rank correlation coefficient, 0.145 ( $\mathrm{P}=0.29000)$ (Figure 5A). The initial value of CRP-albumin ratio was not correlated with TTF using MIC, 0.260, and Spearman's rank correlation coefficient, $-0.084(\mathrm{P}=0.54300)$ (Figure 5B).

Excluding patients with discontinuation of ICIs due to adverse events or ongoing ICI treatment, the correlation between TTF and IRP, the initial NLR or the initial CRPalbumin ratio was not significant in only patients with discontinuation due to tumor progression (Figure 6). Because some patients with discontinuation of ICIs due to adverse events have longer TTF, excluding these patients in addition 


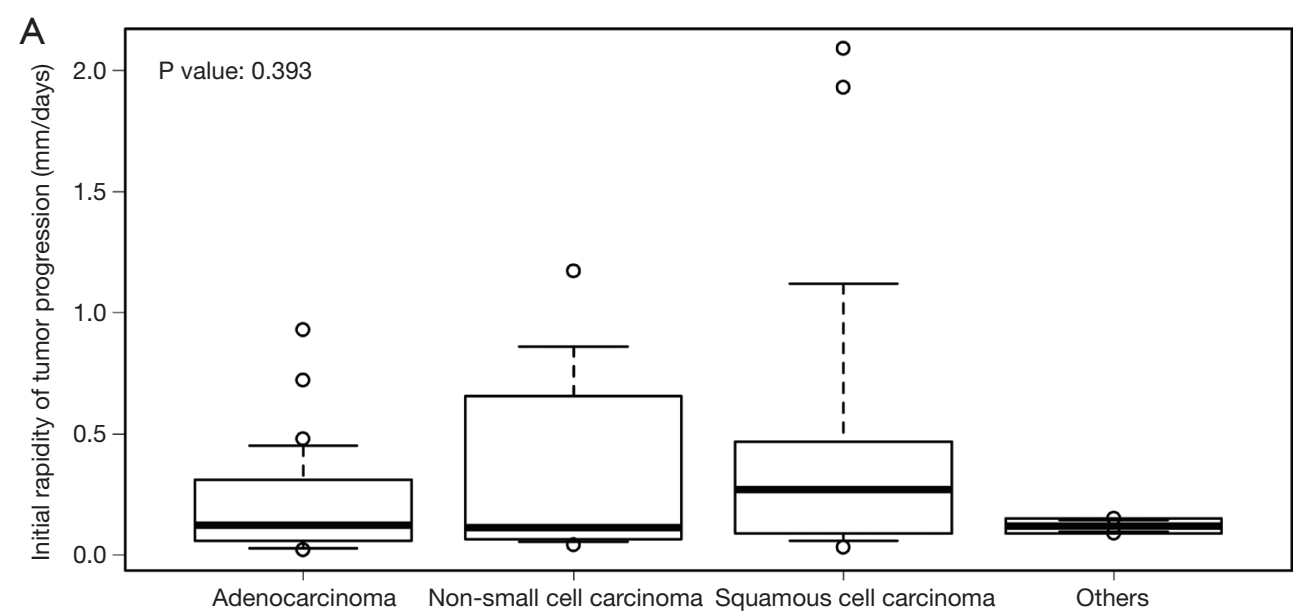

B $\quad$ MIC $=0.234$

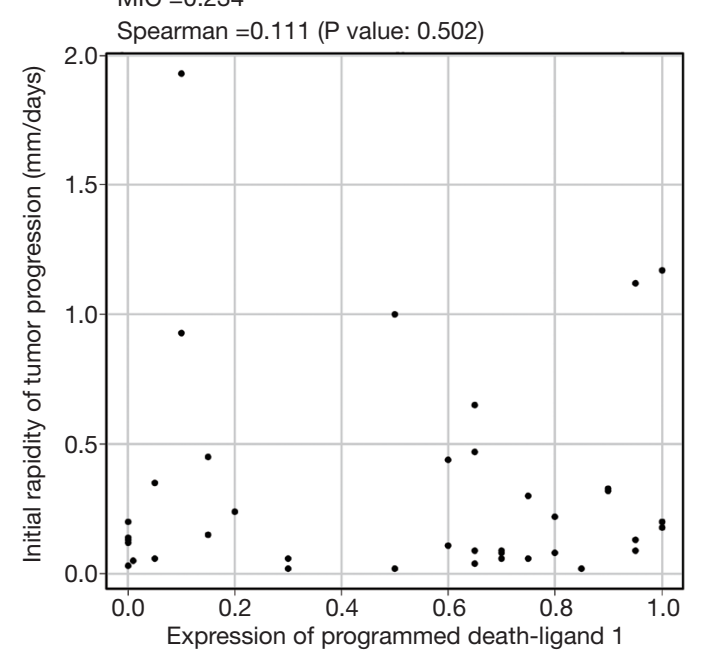

Figure 3 Initial rapidity of tumor progression according to histology of lung cancer or expression of programmed death-ligand 1. (A) Box plot showing initial rapidity of tumor progression in each histology of lung cancer. (B) Scatter plot showing a correlation between initial rapidity of tumor progression and expression of programmed death-ligand 1. They revealed no correlation. MIC, maximal information coefficient; Spearman, Spearman's rank correlation coefficient.

to patients with ongoing ICI treatment might omit patients who were able to have benefit by ICI treatment for TTF.

\section{Discussion}

In this research, we evaluated the tumor growth rate, IRP, as a prognostic factor of ICI treatment, comparing it with other previously reported prognostic factors: ITS, initial NLR, and the initial value of CRP-albumin ratio. Because some of these factors might have non-linear correlations with prognosis, we needed to use scatter plots rather than survival curves for estimation; however, at present, there is no standard non-linear correlation coefficient. The correlation of each factor with TTF was evaluated by drawing scatter plots, and MIC, a non-parametric statistic, followed by Spearman's rank correlation coefficient was calculated. We adopted MIC, as described in the language Python or R, because it has been developed to show the distribution of non-linearly related variables on a scatter plot. Although, at present, no commonly used methods for calculating the correlation of clinical factors have been approved for non-linear distributions, MIC has successfully been applied to genome-wide association studies as a nonparametric statistic (18). In this investigation, MIC showed 

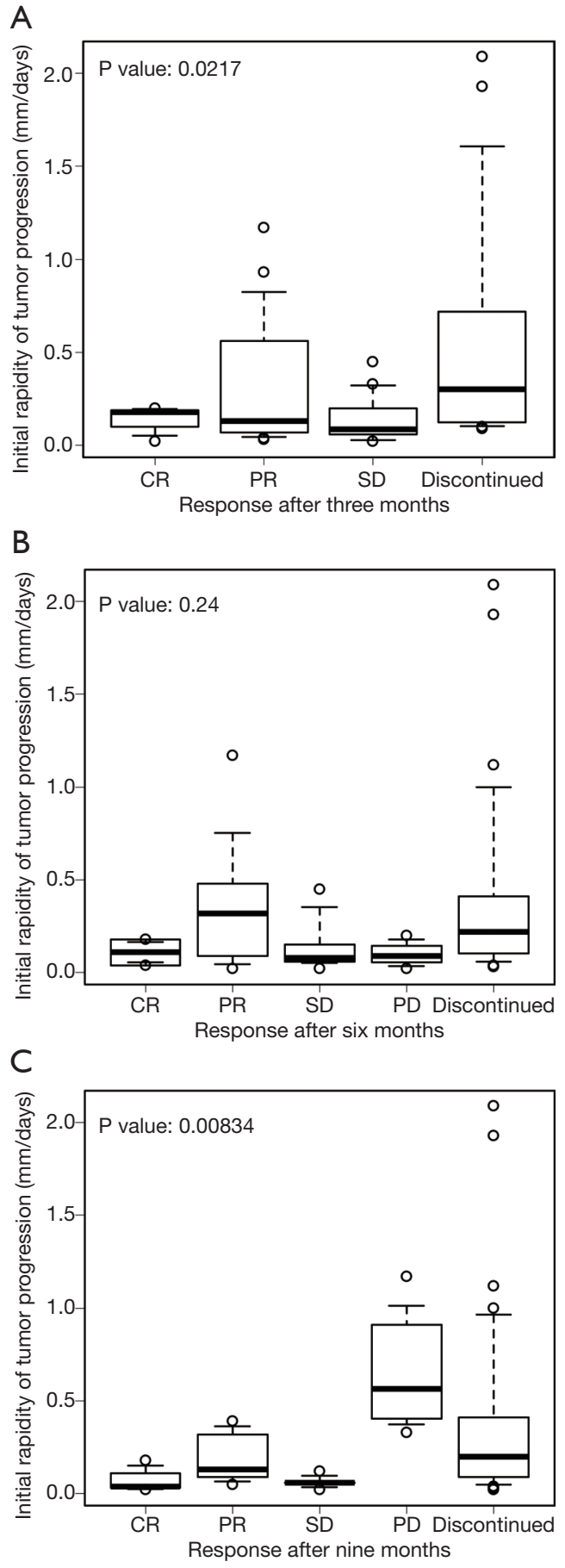

Figure 4 Box plots showing initial rapidity of tumor progression in each response to immune-checkpoint inhibitors (ICI) at 3 (A), 6 (B), and $9(\mathrm{C})$ months after ICI treatment. CR, complete response; PR, partial response; $\mathrm{SD}$, stable disease; $\mathrm{PD}$, progression disease.
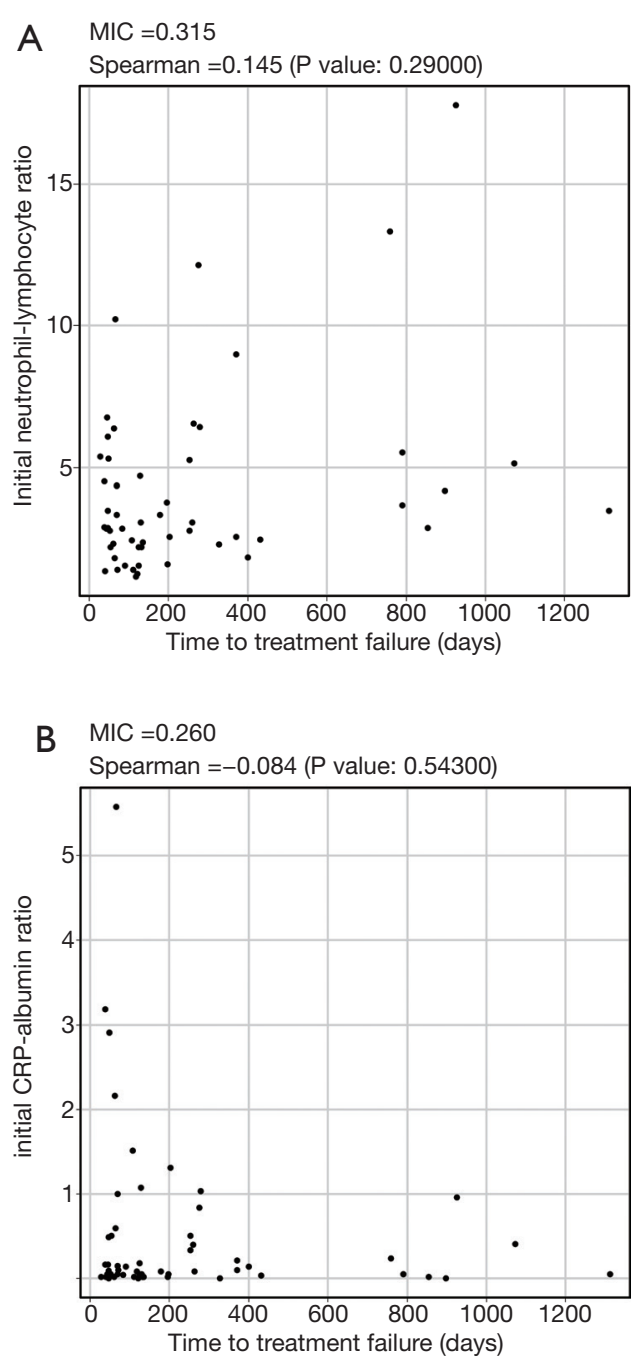

Figure 5 Scatter plot showing a correlation between time to treatment failure (TTF) and previously-published- and clinicalprognostic factors. (A) Correlation between initial neutrophillymphocyte ratio and TTF. (B) Correlation between initial CRPalbumin ratio and TTF. MIC, maximal information coefficient; Spearman, Spearman's rank correlation coefficient; CRP, C-reactive protein.

that there were correlations of the initial NLR, which is an already reported prognostic factor, and IRP with TTF, before we examined whether the correlations were linear.

The scatter plots showed NLR and TTF were positively correlated in ICIs-treated patients, although NLR has recently been reported to be negatively correlated with the 
A $\quad \mathrm{MIC}=0.223$

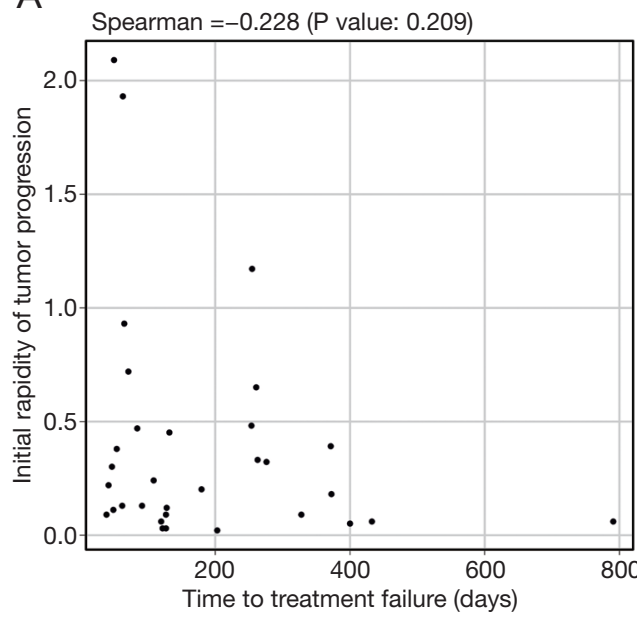

C $\quad \mathrm{MIC}=0.221$

Spearman $=0.152(P$ value: 0.405$)$

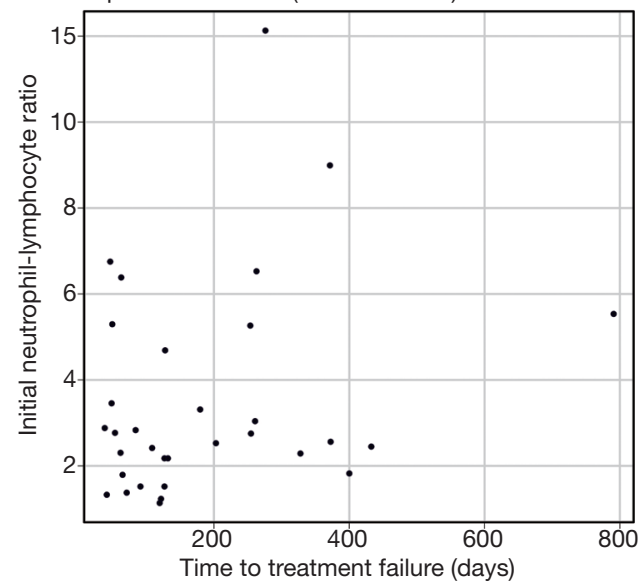

B $\quad$ MIC $=0.265$

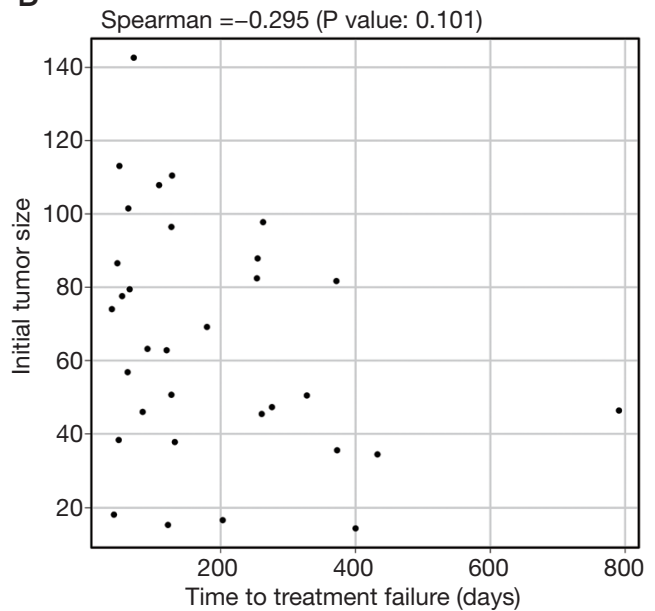

D $\quad \mathrm{MIC}=0.235$

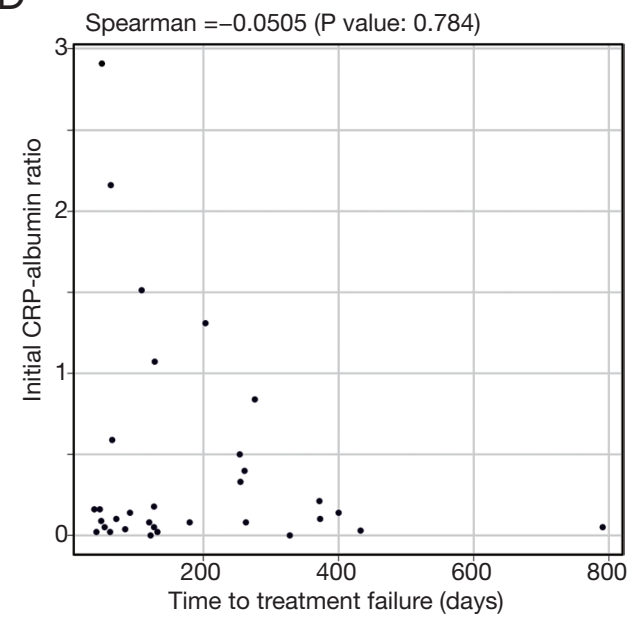

Figure 6 Scatter plots showing a correlation between time to treatment failure (TTF) and initial rapidity of tumor progression (A), initial tumor size (B), initial neutrophil-lymphocyte ratio (C), and initial CRP-albumin ratio (D) in only patients with discontinuation of immunecheckpoint inhibitor (ICI) due to tumor progression, excluding patients with discontinuation of ICI due to adverse events or ongoing ICI treatment. MIC, maximal information coefficient; Spearman, Spearman's rank correlation coefficient; CRP, C-reactive protein.

effect of ICIs (16). This controversial result is attributed to the lack of accepted measures for evaluating each prognostic predictor of ICIs treatment. Katsurada et al. reported the ITS as a useful predictor (12), but tumor size and the rate of tumor growth are not necessarily linearly correlated, which indicates that time-sequential observation of tumor progression before ICI treatment might be useful. When we examined the rate of tumor growth before ICI treatment, and evaluated its correlation with TTF, MIC proved to be weak; furthermore, Spearman's rank correlation coefficient supported this correlation more than the correlation with tumor size. In addition, even though previously-reported prognostic factors did not regress linearly on TTF, our study suggested that only the rate of tumor growth had negatively correlated with TTF in a linear manner.

From our results, ICI treatment of acutely progressive tumors is less beneficial; however, our investigation has the following limitations. First, this investigation was a small-scale retrospective study at a designated regional cancer treatment hospital in Japan; therefore, we will set up an independent cohort to validate the results. Secondly, because 4 of 55 patients continued ICI therapy after the end of the follow-up period, the TTF of these patients might blur the results. Since ICIs could give extremely long 
survival, excluding these patients would result in omitting long-term survivors treated with ICI; therefore, we included their data up to the end of the follow-up in the scatter plot. Thirdly, tumor size and response to ICIs were measured on two-dimensional CT scans according to the RECIST guideline. Three-dimensional measurement may improve the accuracy of the results. Fourthly, $40 \%$ of patients received nivolumab; therefore, our results may be affected by the first-released ICI.

The predictors of clinical efficacy of ICIs are yet to be determined owing to the lack of consistent results and differences in procedures between different researchers. Our investigation revealed the correlation between the rate of tumor growth and the therapeutic effect of ICIs using a novel non-parametric test, MIC, and Spearman's rank correlation coefficient. The MIC score presented a weak correlation between the rate of tumor growth and TTF, and they regressed linearly. This result suggests that the effect of ICIs is less beneficial in tumors with an acutely progressive feature in their inherent properties.

\section{Acknowledgments}

Funding: None.

\section{Footnote}

Reporting Checklist: The authors have completed the STROBE reporting checklist. Available at https://dx.doi. org/10.21037/jtd-21-774

Data Sharing Statement: Available at https://dx.doi. org/10.21037/jtd-21-774

Peer Review File: Available at https://dx.doi.org/10.21037/ jtd-21-774

Conflicts of Interest: All authors have completed the ICMJE uniform disclosure form (available at https://dx.doi, org/10.21037/jtd-21-774). Akihiko Gemma reports receiving honoraria from Bristol-Myers Squibb K.K, Chugai Pharmaceutical Co. Ltd., Daiichi Sankyo Co. Ltd., Kyorin Pharmaceutical Co., Ltd., AstraZeneka K.K., Ono Pharmaceutical Co. Ltd., Eisai Co. Ltd., Nippon Boehringer Ingelheim Co. Ltd., Pfizer Japan Inc. and TAIHO Pharmaceutical Co. Ltd. The other authors have no conflicts of interest to declare.
Ethical Statement: The authors are accountable for all aspects of the work in ensuring that questions related to the accuracy or integrity of any part of the work are appropriately investigated and resolved. The study was conducted in accordance with the Declaration of Helsinki (as revised in 2013). The study was approved by the ethics board of Saitama Medical Center, Saitama Medical University (application number: 2179) and individual consent for this retrospective analysis was waived.

Open Access Statement: This is an Open Access article distributed in accordance with the Creative Commons Attribution-NonCommercial-NoDerivs 4.0 International License (CC BY-NC-ND 4.0), which permits the noncommercial replication and distribution of the article with the strict proviso that no changes or edits are made and the original work is properly cited (including links to both the formal publication through the relevant DOI and the license). See: https://creativecommons.org/licenses/by-nc-nd/4.0/.

\section{References}

1. Fitzmaurice C, Akinyemiju TF, Al Lami FH, et al. Global, regional, and national cancer incidence, mortality, years of life lost, years lived with disability, and disability-adjusted life-years for 29 cancer groups, 1990 to 2016: A systematic analysis for the global burden of disease study. JAMA Oncol 2018;4:1553-68.

2. Sakai K, Kuramoto J, Kojima A, et al. Validation of prognostic impact of number of extrathoracic metastases according to the eighth TNM classification: A singleinstitution retrospective study in japan. Int J Clin Oncol 2019;24:1549-57.

3. Shepherd FA, Dancey J, Ramlau R, et al. Prospective randomized trial of docetaxel versus best supportive care in patients with non-small-cell lung cancer previously treated with platinum-based chemotherapy. J Clin Oncol 2000;18:2095-103.

4. Brahmer J, Reckamp KL, Baas P, et al. Nivolumab versus docetaxel in advanced squamous-cell non-small-cell lung cancer. N Engl J Med 2015;373:123-35.

5. Borghaei H, Paz-Ares L, Horn L, et al. Nivolumab versus docetaxel in advanced nonsquamous non-small-cell lung cancer. N Engl J Med 2015;373:1627-39.

6. Reck M, Rodriguez-Abreu D, Robinson AG, et al. Pembrolizumab versus chemotherapy for PD-L1positive non-small-cell lung cancer. N Engl J Med 
2016;375:1823-33.

7. Gandhi L, Rodríguez-Abreu D, Gadgeel S, et al. Pembrolizumab plus chemotherapy in metastatic nonsmall-cell lung cancer. N Engl J Med 2018;378:2078-92.

8. Paz-Ares L, Luft A, Vicente D, et al. Pembrolizumab plus chemotherapy for squamous non-small-cell lung cancer. $\mathrm{N}$ Engl J Med 2018;379:2040-51.

9. Rizvi H, Sanchez-Vega F, La K, et al. Molecular determinants of response to anti-programmed cell death (PD)-1 and anti-programmed death-ligand 1 (PD-L1) blockade in patients with non-small-cell lung cancer profiled with targeted next-generation sequencing. J Clin Oncol 2018;36:633-41.

10. Chang L, Chang M, Chang HM, et al. Microsatellite instability: A predictive biomarker for cancer immunotherapy. Appl Immunohistochem Mol Morphol 2018;26:e15-21.

11. Kagamu H, Kitano S, Yamaguchi O, et al. CD4(+) T-cell immunity in the peripheral blood correlates with response to anti-PD-1 therapy. Cancer Immunol Res 2020;8:334-44.

12. Katsurada $M$, Nagano T, Tachihara $M$, et al. Baseline

Cite this article as: Sakai $\mathrm{K}$, Kuramoto J, Nishimura $\mathrm{H}$, Kuwabara Y, Kojima A, Sasaki-Toda M, Ogawa-Kobayashi Y, Kikuchi S, Hirata Y, Mikami-Saito Y, Mikami S, Kyoyama H, Moriyama G, Gemma A, Uematsu K. Initial rapidity of tumor growth as a prognostic factor for the therapeutic effect of immune-checkpoint inhibitors in patients with non-small cell lung cancer: evaluation for linear and non-linear correlation. J Thorac Dis 2021;13(8):4903-4914. doi: 10.21037/jtd-21-774 tumor size as a predictive and prognostic factor of immune checkpoint inhibitor therapy for non-small cell lung cancer. Anticancer Res 2019;39:815-25.

13. Reshef DN, Reshef YA, Finucane HK, et al. Detecting novel associations in large data sets. Science 2011;334:1518-24.

14. Kanda Y. Investigation of the freely available easy-touse software 'EZR' for medical statistics. Bone Marrow Transplant 2013;48:452-8.

15. Norton L. A Gompertzian model of human breast cancer growth. Cancer Res 1988;48:7067-71.

16. Bagley SJ, Kothari S, Aggarwal C, et al. Pretreatment neutrophil-to-lymphocyte ratio as a marker of outcomes in nivolumab-treated patients with advanced non-small-cell lung cancer. Lung Cancer 2017;106:1-7.

17. Inoue T, Tamiya M, Tamiya A, et al. Analysis of early death in Japanese patients with advanced non-small-cell lung cancer treated with nivolumab. Clin Lung Cancer 2018;19:e171-6.

18. Yang D, Liu H. Maximal information coefficient applied to differentially expressed genes identification: A feasibility study. Technol Health Care 2019;27:249-62. 